Editor's Note: These short, critical reviews of recent papers in the Journal, written exclusively by graduate students or postdoctoral fellows, are intended to summarize the important findings of the paper and provide additional insight and commentary. For more information on the format and purpose of the Journal Club, please see http://www.jneurosci.org/misc/ifa_features.shtml.

\title{
Micromanaging Oligodendrocyte Differentiation by Noncoding RNA: Toward a Better Understanding of the Lineage Commitment Process
}

\author{
James Butcher, Houssein Abdou, ${ }^{*}$ Katy Morin, ${ }^{*}$ and Yubing Liu ${ }^{*}$ \\ Ottawa Institute of Systems Biology and Department of Biochemistry, Microbiology, and Immunology, University of Ottawa, Ottawa, Ontario K1H 8M5, \\ Canada \\ Review of Lau et al. (http://www.jneurosci.org/cgi/content/full/28/45/11720)
}

Differentiation is one of the most complex and paradoxical cellular processes that multicellular organisms undergo (Chen and Daley, 2008). Although each cell in a multicellular organism contains the same genome, genetic information is processed differently once cells terminally differentiate. This phenomenon ultimately produces the various tissues and specialized cells required for higher eukaryotic function. To fully comprehend this process, we must unravel the complex regulatory mechanisms that control cell type commitment and differentiation (Chen and Daley, 2008). A comprehensive understanding of these processes would yield tremendous dividends, as both brain repair after injury and stem cell therapy rely on the ability to modulate the body's natural ability to self-renew and differentiate into various cell types (Corbin et al., 2008). The discovery of multipotential stem and progenitor cells postnatal has raised new hopes for therapeutic strategies aimed at directing these rare cells toward re-

\footnotetext{
Received Dec. 16, 2008; revised March 13, 2009; accepted March 16, 2009. We acknowledge Drs. Daniel Figeys and Steffany Bennett for their encouragement and support

*H.A., K.M., and Y.L. contributed equally to this work.

Correspondence should be addressed to James Butcher, Ottawa Institute of Systems Biology, Department of Biochemistry, Microbiology, and Immunology, University of Ottawa, 451 Smyth Road, Ottawa, 0N K1H 8M5, Canada. E-mail:jbut076@uottawa.ca.

DOI:10.1523/JNEUROSCI.5976-08.2009

Copyright $\odot$ 2009 Society for Neuroscience $\quad 0270-6474 / 09 / 295365-02 \$ 15.00 / 0$
}

quired lineages (astrocytes, oligodendrocytes, and neurons) and promoting appropriate subtype specification.

MicroRNAs (miRNAs) are small noncoding RNAs increasingly recognized as major players in post-transcriptional gene regulation (Ouellet et al., 2006; Chen and Daley, 2008). These regulators function by base pairing to their target mRNAs to either repress translation or enhance mRNA degradation. miRNAs are believed to recognize their targets primarily by the presence of a "seed" region of perfect complementarity present in their target mRNAs. miRNAs are also believed to bind sequences solely in the $3^{\prime}$ untranslated region (UTR) of their target $\mathrm{mR}$ NAs, although this view has been challenged recently (Didiano and Hobert, 2006). miRNAs are normally transcribed as separate transcriptional units, but several have been found to be present in introns, where they are coexpressed with their target immature mRNA. Although much is known about the processing of pre-miRNAs to mature miRNAs, little is known about their actual targets in vivo beyond recent reports implicating miRNAs in the modulation and refinement of embryonic stem cell (ESC) differentiation (Tay et al., 2008). This revelation has reinvigorated the field. Studying miRNAs could open up new paradigms in designing therapeutic targets to both modulate and control cellular differentiation.
Lau et al. (2008) took an important step toward this goal. The authors reported the first in vivo miRNA expression profile in a defined neural precursor cell lineage that directs terminal specification. Using fluorescence activated cell sorting, Lau et al. (2008) isolated oligodendrocyte progenitor cells (OPCs) and oligodendrocyte linage cells (OLs) based on their differential expression of cell surface markers (their Fig. 1A). This allowed for the creation of two homogenous populations of distinct cell lineages. Lau et al. (2008) then determined their miRNA profile using miRNA microarrays. The expression of 98 miRNAs was detected and validated using quantitative PCR. This list of miRNAs included 38 that were located within introns, and the expression of their host genes was also confirmed using microarrays. Many of these miRNAs, such as miR-9 and miR-219, had been previously described as being brain enriched (Lau et al., 2008).

The inherent innovation in this study lies in the subsequent target bias analysis performed by the authors to enrich for functional miRNA targets. This procedure assumes that functional targets will have an expression profile that correlates with their miRNA regulator. This functional enrichment used microarrays to determine gene expression. Predicted miRNA targets were then compiled using an algorithm that predicts miRNA targets based on base pair complementarity be- 
tween the miRNA seed region and the $3^{\prime}$ UTR of the target mRNA (Lewis et al., 2003; Ouellet et al., 2006). The analysis revealed that 16 of the 98 identified miRNAs showed a target bias when the profiles of the OPCs and OLs were compared. This suggested that expression is not only cell type specific, but, more excitingly, "differentiation stage" specific. Additional analysis revealed that 9 of these 16 miRNAs appeared to regulate their targets and hence could be important for guiding oligodendrocyte differentiation.

As proof of principle that identified miRNA's impact mRNA translation to direct cell fate, the authors characterized the effect of miR-9 on target transcripts. PMP22 is a component of myelin and is expressed primarily by Schwann cells in the peripheral nervous system. PMP22 was predicted to be a target of miR-9 based on its $3^{\prime}$ mRNA sequence. However, PMP22 protein has not been previously detected in oligodendrocytes of the CNS, although the PMP22 transcript was present (Dugas et al., 2006; Lau et al., 2008). Lau et al. (2008) fused various fragments of the PMP22 3'UTR to a luciferase reporter construct to determine if $\mathrm{CO}^{-}$ expression of miR- 9 affected luciferase protein levels. In vivo results, as well as in vitro binding assays, demonstrated that miR-9 interacted with two regions of the PMP22 3'UTR, each containing the seed sequence of miR-9. The presence of these seed regions in the $3^{\prime}$ region of the luciferase mRNA reduced protein expression and luminescence in cotransfected HeLa cells. In addition, transient transfection of mature miR-9 in Schwann cells (which normally express only the inactive pre-miRNA of MiR-9) was shown to moderately downregulate PMP22 mRNA and protein levels. These data provided elegant evidence that miRNAs can modulate transcripts as part of the delicate dance toward differentiation and further suggests that miRNAs could be important in maintaining cellular identity once terminal differentiation has been achieved.

Together, this study provides compelling evidence for the presence of miRNA regulation throughout the process of OPC differentiation to OLs. They convincingly show that different miRNAs exhibit preferential expression at specific stages during differentiation. This observation was strengthened by the fact that intronic miRNA expression was correlated with its host gene expression. This suggests that certain miRNAs could be controlled by previously identified mRNA transcription modulators. Post-transcription control could also function by modulating miRNA's maturation process or by affecting
miRNA's degradation rates. Posttranscriptional regulation of miRNAs is illustrated in the case of Schwann cells and miR-9 described above. Schwann cells express unprocessed miR-9 that allows continued expression of PMP22 protein. However, the presence of mature miR-9 reduced PMP22 mRNA and protein levels, demonstrating that miR-9 function is normally repressed in Schwann cells via post-transcriptional mechanisms.

However, the work of Lau et al. (2008) also spotlights a key problem in miRNA functional analysis: the difficulty in identifying potential miRNA targets (Ouellet et al., 2006). Most of the bioinformatic programs available rely solely on matching the miRNA seed region to a complementary region present in the 3'UTR of target genes (Ouellet et al., 2006). However, these programs are limited by incomplete knowledge of the structural determinants governing miRNAtarget interactions. There is now evidence that miRNAs can regulate genes by binding to regions outside the 3'UTR (Tay et al., 2008). There are also conflicting views on whether miRNAs can regulate genes by binding to an imperfect match to their seed region (Didiano and Hobert, 2006). Furthermore, many, if not all, bioinformatic algorithms only take classical Watson-Crick base pairing into consideration when locating potential miRNA targets (Didiano and Hobert, 2006; Ouellet et al., 2006). This ignores the other base paring that is possible in RNA complexes as well as the complex three-dimensional structures that RNAs form in vivo. To date, there are currently no programs available that are able to consider all these additional possibilities when predicting miRNA-binding sites (Ouellet et al., 2006). As such, the current technologies used to locate miRNA-binding sites are almost certain to incorrectly identify a significant number of potential sites and to miss many actual binding sites. Thus, any genome-wide bioinformatic mapping of potential miRNA-regulated genes is fraught with error, and the results should be scrutinized closely before drawing conclusions. Lau et al. (2008) provided a salient example of this technical limitation. Their bioinformatic analysis identified a single potential binding site for miR-9 in the $3^{\prime}$ UTR of PMP22 mRNA. However, deletion of the predicted binding site did not relieve miR-9 inhibition. Closer examination of the 3'UTR sequence revealed another site that contained the predicted seed region of miR-9 repeated in tandem but had a lower overall region of complementarity outside the seed region. Lau et al. (2008) demonstrated that either region was capable of in- teracting with miR-9 through in vitrobinding assays and in vivo luciferase assays. This highlights the need to confirm predicted miRNA interactions as the current bioinformatic algorithms undergo refinement. Many reported miRNA-regulated genes may actually be artifacts, whereas numerous real miRNA-regulated genes have likely been missed.

Recent work in ESCs had identified miRNAs to be key modulators of differentiation (Tay et al., 2008) but did not assess whether these miRNAs were differentially expressed during the process of differentiation. The report by Lau et al. (2008) not only identified miRNAs that are specific for oligodendrocytes but also miRNAs that were specific to differentiation. This opens up new therapeutic targets and strategies to combat various differentiation-linked diseases. The discovery of oligodendrocytespecific miRNAs allows for the possibility that other neuronal cell types could also be partially specified by the presence or absence of miRNAs (Chen and Daley, 2008; Corbin et al., 2008). The discovery of these miRNA modulators could allow for the remodeling of cellular progenitor differentiation toward certain lineages as needed. The ability to fine tune and control this process could lead to countless discoveries and treatments for a wide variety of diseases, emphasizing the importance of "unbiased" network analysis as part of the basic science necessary to realize this goal.

\section{References}

Chen L, Daley GQ (2008) Molecular basis of pluripotency. Hum Mol Genet 17:R23-R27.

Corbin JG, Gaiano N, Juliano SL, Poluch S, Stancik E, Haydar TF (2008) Regulation of neural progenitor cell development in the nervous system. J Neurochem 106:2272-2287.

Didiano D, Hobert O (2006) Perfect seed pairing is not a generally reliable predictor for miRNA-target interactions. Nat Struct Mol Biol 13:849-851.

Dugas JC, Tai YC, Speed TP, Ngai J, Barres BA (2006) Functional genomic analysis of oligodendrocyte differentiation. J Neurosci 26:10967-10983.

Lau P, Verrier JD, Nielsen JA, Johnson KR, Notterpek L, Hudson LD (2008) Identification of dynamically regulated microRNA and mRNA networks in developing oligodendrocytes. J Neurosci 28:11720-11730.

Lewis BP, Shih IH, Jones-Rhoades MW, Bartel DP, Burge CB (2003) Prediction of mammalian microRNA targets. Cell 115:787-798.

Ouellet DL, Perron MP, Gobeil LA, Plante P, Provost $P$ (2006) MicroRNAs in gene regulation: when the smallest governs it all. J Biomed Biotechnol 2006:69616.

Tay Y, Zhang J, Thomson AM, Lim B, Rigoutsos I (2008) MicroRNAs to Nanog, Oct4 and Sox2 coding regions modulate embryonic stem cell differentiation. Nature 455:1124-1128. 